Cipango
Cipango

$22 \mid 2015$

Du particulier et de l'universel

\title{
Topoï de la mort violente et corps psychotique dans Yūkoku de Mishima Yukio
}

Topoi of Violent Death and the Psychotic Body in Mishima Yukio's Yūkoku

\section{Thomas Garcin}

\section{OpenEdition}

1 Journals

Édition électronique

URL : https://journals.openedition.org/cipango/3065

DOI : 10.4000/cipango.3065

ISSN : 2260-7706

\section{Éditeur}

INALCO

\section{Édition imprimée}

Date de publication : 1 janvier 2015

ISSN : 1164-5857

Référence électronique

Thomas Garcin, "Topoï de la mort violente et corps psychotique dans Yūkoku de Mishima Yukio », Cipango [En ligne], 22 | 2015, mis en ligne le 12 février 2019, consulté le 09 avril 2022. URL : http:// journals.openedition.org/cipango/3065 ; DOI : https://doi.org/10.4000/cipango.3065

\section{(c) (i) (9)}

Cipango est mis à disposition selon les termes de la Licence Creative Commons Attribution - Pas d'Utilisation Commerciale 4.0 International. 
VARIA 



\title{
Topö̈ de la mort violente et corps psychotique dans Yükoku de Mishima Yukio ${ }^{1}$
}

\author{
Topoi of Violent Death and the Psychotic \\ Body in Mishima Yukio's Yükoku
}

Thomas GARCIN

Pembroke College, University of Oxford

\section{Introduction}

Publiée pour la première fois dans la revue Shōsetsu chūō kōron 小説中央公論 en janvier 1961, la nouvelle Yükoku 憂国 (Patriotisme) décrit le suicide du lieutenant Takeyama Shinji 武山信二 et de son épouse Reiko 麗子 pendant les incidents du 26 février 1936 (ni-niroku-jiken二・二六事件) ${ }^{2}$. Partageant l'amitié et les convictions politiques des rebelles, Shinji n’a toutefois pas été invité à participer

2. Le 26 février 1936 de jeunes officiers mutins, à la tête d'environ 1400 soldats, ont tenté un coup d'État en vue de renverser un gouvernement qu'il jugeait corrompu et « vendu à l'étranger ». Ils espéraient recevoir le soutien de l'Empereur mais celui-ci se déclara prêt à diriger lui-même la répression. La révolte fut rapidement matée et ses leaders exécutés. Ben-Ami Shillony, Revolt in Japan: The Young Officers and the February 26, 1936 Incident, Princeton, Princeton University Press, 1973. 
198 Cahiers d'études japonaises n²2

au coup d'État. De retour chez lui à l'occasion d'une courte permission, il choisit, afin de concilier l'obéissance qu'il doit à ses supérieurs avec ses convictions intimes, d'accomplir le suicide rituel par éventrement (seppuku 切腹). Sa femme propose de l'accompagner. Le couple se livre à un dernier coït solennel avant de se donner la mort. Ce récit est inspiré d'un fait divers qui a eu lieu en marge de la tentative de coup d'État. Dans la nuit du 28 février 1936, le lieutenant Aoshima Kenkichi 青 島健吉du Bataillon de logistique de la Garde impériale, un ami des officiers rebelles qui n'était pas personnellement impliqué, s'éventra à son domicile de Setagaya, accompagné de sa jeune épouse ${ }^{3}$. L'arrière-plan idéologique de la nouvelle et le motif du seppuku ont amené la plupart des commentateurs à étudier ce texte sous un angle thématique et/ou biographique. Ce récit annonce en effet l'engagement croissant de l'auteur en faveur de l'ultranationalisme dans le cours des années 1960. Dans une perspective téléologique, il pourrait aussi être perçu comme le présage de son propre éventrement. La nouvelle nous renvoie enfin à toute une série de thématiques très largement présentes dans l'ensemble de son œuvre : refus du principe de réalité, quête de la transcendance, pulsion de mort, sadomasochisme, narcissisme, etc. L'importance que les obsessions politiques et les fétiches de l'auteur ont pu avoir dans la rédaction de ce texte est donc indéniable. Il nous semble qu'une attention plus grande aurait toutefois mérité d'être portée à la place, dans le texte, des conventions littéraires liées à la représentation de la mort volontaire au Japon. Mishima ne se contente pas d'investir par l'écriture un fantasme d'ordre personnel. À travers le motif de l'éventrement et du suicide des amants, il brode aussi sur des « scénarios intertextuels » $(\mathrm{Eco})^{4}$ très présents dans la littérature nationale. Loin de prétendre s'affranchir des topoï que véhicule, en tant que fait culturel majeur, la représentation de la mort volontaire, Mishima les exploite très largement. Des stéréotypes qui pourraient sembler à certains égards incompatibles se heurtent, s'éclairent mutuellement et un surcroît de sens et de littérarité naît de leur collision. L'objet de cette étude sera de montrer quels sont les topoï de la mort volontaire présents dans Yükoku et de voir comment ils

\section{Ibid., p. 191.}

4. Umberto Eco, Lector in Fabula - Le rôle du lecteur ou la coopération interprétative dans les textes narratifs, Paris, Grasset, 1985 (1979), p. 108. 
s'articulent les uns aux autres. Nous proposerons, à l'issue de cette analyse, une relecture de la nouvelle à travers la grille interprétative du corps psychotique qui offre un dénominateur commun à ces représentations hétéroclites.

\section{L’imaginaire épique : mort monumentale et corps héroïque}

La lecture de la nouvelle Yükoku s'ouvre sur un premier chapitre déroutant qui résume toutel'intrigue en japonais classique, sur un ton à la fois sobre et emphatique. En supprimant tout suspens narratif, le narrateur tend ici à attirer l'attention du lecteur sur la forme même du textes. Il donne d'autre part au récit une touche désuète évoquant les genres archaïques, imprégnées d'oralité, et transmis par un intermédiaire (conteur, récitant, aède). Le décalage manifeste de cette langue et de ce type de contrat de lecture avec l'attente des lecteurs contemporains suggère un usage délibéré des stéréotypes. L'auteur joue ici avec des conventions littéraires surannées qui peuvent notamment évoquer l'épopée ${ }^{6}$ :

Le vingt-huit février mil neuf-cent trente-fix (foit au troisième jour de l'incident du vingt-fix février), dans la pièce de huit

5. Hasegawa Izumi 長谷川泉, «Yükoku, Tōka no kiku, Eirei no koe ni furete 》『 憂国』、『十日の菊』、『英霊の聲』に触れて (Au sujet de Patriotisme, Le Chrysanthème du dernier jour et La Voix des mânes hérö̈ques), Gendai no esupuri 現代の エスプリ, nº 48, février 1971, p. 138-157.

6. La traduction anglaise - Patriotism (1966), traduit du japonais par Geoffrey W. SARGENT (New York, The New Directions Pearls, 2010) - et par conséquent la traduction française qui en est tirée - Patriotisme dans La Mort en été (1983), traduit de l'anglais par Dominique Aury (Paris, Gallimard, 1983, p. 165-202) - ne cherche pas à rendre le japonais classique du premier chapitre. Il est vrai qu' il est difficile de trouver un équivalent dans la mesure où l'anglais et le français moderne se forment plus tôt que le japonais moderne. Utiliser des archaïsmes linguistiques (en français les anciennes terminaisons, par exemple, de l'imparfait) donnerait ainsi au chapitre introductif une dimension burlesque qui nous semble absente du texte d'origine. Nous avons essayé de trouver un compromis en reportant l'archaisme linguistique sur des éléments typographiques : $s$ long noté $f$ et esperluette (\&), derniers reliquats de l'ancien français qui disparaissent à la fin du XVIIIe siècle. Nous nous sommes, pour ce faire, inspiré des gazettes du XVIIIe siècle disponibles en ligne : http://www.gazettes 18e.fr/. 
nattes de fon domicile - fixième périmètre du quartier Aoba, arrondissement de Yotsuya - le lieutenant Takeyama Shinji du premier bataillon d'infanterie de la Garde Impériale, tourmenté au plus haut point fuite à l'engagement de fes amis aux côtés des infurgés, \& profondément indigné par les circonftances qui mettaient l'armée impériale en prife avec fes propres troupes, exécuta, de son fabre de foldat, l'éventrement rituel ; fon époufe Reiko, fidèle à fon époux, retourna femblablement fon poignard contre elle-même. Le teftament du lieutenant se limitait à cette simple phrafe : «Que vive éternellement l'Armée impériale ». Son époufe follicitait l'indulgence de fes parents pour l'ingratitude dont elle fe rendait coupable en les précédant dans la mort, ajoutait que le jour était arrivé auquel elle était « destinée en tant que femme de foldat », \& cætera. Les derniers inftants de ce couple vertueux furent véritablement de nature à faire pleurer les plus terribles de nos dieux. Précifons que le lieutenant avait fêté fes trente et un ans, que fon épouse comptait vingt-trois printemps \& que fix mois ne f'étaient pas écoulés depuis la célébration de leurs noces ${ }^{7}$.

L'une des principales fonctions de l'épopée, note Judith Labarthe, est de « rappeler [les] exploits » des héros combattants et de «faire retentir leurs

7. Yükoku, in Eirei no koe 英霊の聲 (La voix des mânes héroïques), chapitre 1, p. 75. Nous utilisons la version de poche des éditions Kawade Shobō 河出書房 réunissant, sous le titre Eirei no koe, les textes Eirei no koe, Tōka no kiku 十日の菊 (Le chrysanthème du dixième jour, 1963) et Yükoku. Ce triptyque dédié aux incidents du 26 février 1936 est accompagné d'une postface Ni-ni-roku jiken to watashi 二・二六事件と私 (Les incidents du 26 février et moi) dans laquelle Mishima évoque son intérêt pour cet évènement historique. Le paratexte et le cotexte appuient ici fortement la portée idéologique du récit. Il est à noter que quelques corrections mineures ont été apportées au texte par rapport à la version originale parue en janvier 1961 (ibid., p. 260-261). Par commodité nous nous contenterons, lorsque nous citerons des passages de Yükoku dans la suite de cette étude, de noter les numéros de chapitre et de pages. 
noms $»^{8}$. À travers ce premier chapitre, Yūkoku se présente de la même façon comme une sorte de tombeau érigé en l'honneur du lieutenant Takeyama Shinji et de son épouse Reiko. Toute la nouvelle se déploie, à partir du seuil inaugural du premier chapitre, comme une longue hypotypose, figure privilégiée des genres épiques qui « consiste à mettre sous les yeux une scène ${ }^{9}$. Le narrateur non seulement montre, mais se montre comme celui qui montre. Dans la suite de la nouvelle, il ne cesse d'ailleurs, fût-ce de façon plus discrète, de laisser entrevoir la position depuis laquelle il s'exprime, prenant ainsi à contre-pied, comme l'a noté Satō Hideaki ${ }^{10}$, la norme du roman réaliste post-flaubertien.

Outre la situation énonciative du texte, c'est le sujet même du récit - la mort exemplaire d'un jeune couple héroïque - qui est susceptible d'évoquer les genres épiques. Dans le chapitre introductif, le narrateur de Yükoku note que ce sont « les derniers moments » du couple qui sont exemplaires. Le terme «saigo » (écrits avec les sinogrammes 最期) choisi par le narrateur n'est pas anodin. Il apparaît fréquemment dans les épopées médiévales japonaises (gunkimono 軍記物) pour désigner les fins exemplaires des guerriers. Dans le Heike monogatari 平家物語 (Le dit des Heike), il est ainsi utilisé à neuf reprises en tant que titre d'épisodes relatant la mort héroïque de combattants valeureux. Ce motif, fait remarquer Daniel Struve, est « étroitement lié à la représentation de la condition des guerriers pour lesquels l'instant de la mort tend à prendre une importance décisive et en vient à symboliser la réussite ou l'échec d'une vie $\gg^{11}$. Les morts spectaculaires qui jalonnent les gunkimono se présentent comme des démonstrations, des preuves par l'exemple de la valeur de celui qui s'y oblige. D'une extrême violence et nécessitant un grand courage et une parfaite maîtrise de soi, le seppuku est l'un des types de

8. Judith Labarthe, L'Épopée, Paris, Armand Colin, 2007, p. 321.

9. Ibid., p. 339 .

10. SATō Hideaki 佐藤秀明, Mishima Yukio no bungaku 三島由紀夫の文学 (La littérature de Mishima), Tōkyō, Shironsha 試論社, 2009, p. 295-302.

11. Daniel Struve, « Récits de fin exemplaire - narration épique et scénicité », in Claire-Akiko Brisset, Arnaud Brotons, et Daniel Struve (dir.), De l'épopée au Japon: Narration épique et théâtralité dans Le Dit des Heike, Paris, Riveneuve, 2011, p. 113. 
202 Cahiers d'études japonaises n²2

saigo les plus fréquents dans les gunkimono, notamment dans les textes les plus tardifs comme le Gikeiki 義経記 (La chronique de Yoshitsune) et surtout le Taiheiki 太平記 (Chronique de la Grande Paix). « La légende du seppuku, note ainsi Andrew Rankin dans l'ouvrage qu'il consacre à cette pratique, s'enracine dans les textes épiques $\gg{ }^{12}$.

À l'instar des héros épiques, le personnage du lieutenant dans Yükoku considère son éventrement comme un moyen de s'absolutiser dans l'acte, de coïncider pour l'éternité avec son statut de soldat destiné à mourir de façon glorieuse sur le champ de bataille :

C'était une mort de même nature et de même intensité que celle que l'on trouvait sur le champ de bataille, une mort qui nécessitait une détermination semblable à celle du guerrier au moment $\mathrm{du}$ combat décisif. Il allait se montrer à sa femme tel qu'au front ${ }^{13}$.

La volonté d'exhiber sa mort correspond aussi au modèle épique. Le motif du saigo implique en effet à la fois une exigence éthique, un rapport du guerrier à un code de valeurs et une logique d'autocélébration narcissique, le souci aigu de l'image qu'on laisse derrière soi. Aussi nécessite-t-elle des spectateurs. Les héros des épopées guerrières médiévales interpellent les ennemis ou les amis qui leur font face et annoncent la dimension exemplaire de la mort qu' ils vont donner en

12. Andrew Rankin, Seppuku: A History of Samurai Suicide, Tōkyō, Kodansha International, 2011, p. 42.

13. Chapitre 4, p. 100. 
spectacle ${ }^{14}$. Cette mort fastueuse est bien évidemment un apanage masculin. Les femmes jouent cependant leur propre partition. Les plus exemplaires n'hésitent pas à suivre le chemin de leur maître et mari ${ }^{15}$. La répartition des rôles dans Yükoku est ainsi similaire à celle des couples exemplaires des gunkimono : au guerrier les splendeurs de la mort somptuaire (sacrifice pour le maître, l'honneur, la patrie, etc.) ; à l'épouse, l'effacement, la mort d'accompagnement discrète dédiée au grand principal marital.

La dimension épique de la nouvelle s'exprime enfin dans la présentation et la description des personnages. Pour peindre ses créatures, le narrateur utilise toujours les mêmes qualificatifs mélioratifs - «beau » (utsukushii 美しい) ${ }^{16}$, «blanc » (shiroi 白い) ${ }^{17}$, « pure » (kiyorakana 清らかな) ${ }^{18}$, « viril » (ririshii

14. Parmi de nombreux autres exemples : au chapitre neuf du Heike monogatari le personnage de Ima.i no Shirō, après avoir appris la mort de son maître, interpelle ses ennemis et saute de son cheval le sabre en bouche. Cf. Le Dit des Heiké, traduit du japonais par René SiefFERT, Lagrasse, Verdier, 2012, p. 574. Selon une logique similaire, dans le Gikeiki, le personnage de Tadanobu exhibe son courage en commettant le seppuku. Cf. Kajihara Masaaki 梶原正昭 (dir.), Gikeiki, Nihon koten bungaku zenshu 日本古典文学全集 (Les œuvres de la littérature classique du Japon, 1971), vol. 31, Tōkyō, Shōgakkan 小学館, 1995, p. 336-337. On pourra aussi consulter la traduction anglaise : Yoshitsune, A Fifteenth-Century Japanese Chronicle, traduit du japonais par Hellen Craig McCullough, Tōkyō, University of Tokyo Press, 1966, p. 205.

15. Dans Le Dit des Heiké, le personnage de Kozaishō se jette à la mer après avoir appris la mort de son mari au combat (op. cit., p. 624-626). Dans le Gikeiki, l'épouse de Yoshitsune affirme avec vigueur sa volonté de suivre son époux dans la mort tandis que celui-ci agonise à ses côtés, après s'être tranché le ventre (op. cit., p. 491 ; pour la traduction anglaise, cf. Hellen Craig McCullough, op. cit., 1966, p. 291).

16. Dix-neuf occurrences : chapitre $2:$ p. 76 (deux occurrences) et p. 77 ; chapitre $3:$ p. 80 , p. 83 , p. 88 (deux occurrences), p. 91 (deux occurrences), p. 92, 93 et p. 94 ; chapitre 4 : p. 98, p. 100 (cinq occurrences) et p. 106.

17. Sept occurrences, uniquement pour décrire Reiko : chapitre 2, p. 76 ; chapitre 3, p. 91, p. 92 (deux occurrences), p. 93 , p. 98 et p. 99.

18. Trois occurrences : chapitre 2, p. 76 et p. 78 ; chapitre 4, p. 100. 
204 Cahiers d'études japonaises n²2

凛々しい, ooshii 雄々しい) ${ }^{19}$, etc. - souvent associés aux mêmes substantifs. Se créent ainsi des structures fixes, qui ne sont pas sans évoquer les épithètes homériques. Le personnage de Reiko se voit, par exemple, qualifiée à trois reprises par le syntagme 《belle épouse » (utsukushii tsuma 美しい妻) ${ }^{20}$. Des expressions telles que «beau visage »(utsukushii kao 美しい顔) ${ }^{21}$, 《 nez d'une forme agréable » (katachi no yoi hana 形のよい鼻) ${ }^{22}$ ou 《doigts blancs » (shiroi yubi 白い指 $)^{23}$ sont aussi répétées à différents endroits du texte. La description des héros relève ainsi de ce que Barthes appelle le « portrait rhétorique » qui, à la différence des portraits réalistes, ne décrit pas les personnages mais les réifie dans leur perfection :

[...] ce portrait-là est purement culturel, renvoyant à la peinture (« faite à peindre ») ou à la mythologie (« la taille de Minerve sous les agréments de Vénus »), ce qui est une bonne manière de les abstraire. Le portrait rhétorique, en effet, quoique parfois assez étendu (car l'auteur ne s'en désintéresse nullement), ne peint rien, ni la chose, ni son effet : il ne fait pas voir (et certainement ne le veut pas) ; il caractérise très peu (parfois la couleur des yeux, des cheveux) ; il se contente de nommer les éléments antinomiques dont chacun est parfait ; et comme cette perfection, en bonne théologie, est l'être même de la chose, il suffit de dire qu'un corps est parfait pour qu'il le soit : la laideur se décrit, la beauté se dit ; ces portraits rhétoriques sont donc vides $[\ldots]^{24}$.

19. Trois occurrences pour ririshii (chapitre 2, p. 76 ; chapitre 3, p. 93 ; chapitre 4, p. 100) et pour ooshii (chapitre 2, p. 76 ; chapitre 3, p. 83 et p. 94).

20. Chapitre 3, p. 83 et chapitre 4, p. 100 (deux occurrences).

21. Chapitre 3, p. 88 et p. 91.

22. Chapitre 2, p. 76 et chapitre 3, p. 91.

23. Chapitre 3, p. 93 et p. 98.

24. Roland Barthes, Sade, Fourier, Loyola, Paris, Éditions du Seuil, 1971, p. 27. 
Même longuement développés, le portrait réduit les personnages à une série de courbes (kyokusen 曲線) et de contours (rinkaku 輪郭) harmonieux ${ }^{25}$. Figure parfaite tracée selon le nombre d'or de la géométrie plastique, le corps des héros incarne en lui-même une sorte d'Idée platonicienne. Adoptant la perspective du lieutenant Takeyama, le narrateur remarque que les courbes du corps de Reiko énoncent « quelque chose comme les proportions exactes de la chair » (nikutai no tadashii kiritsu no yō na mono wo shimeshiteita 肉体の正しい規律のような ものを示していた $)^{26}$.

Comme plusieurs critiques ${ }^{27}$ l'ont fait remarquer, ces êtres tout de surface et dénués de défauts sont plutôt semblables à des statues en mouvement qu'aux êtres de chair et d'os dont le roman réaliste cherche à nous donner l'illusion. Leurs gestes sont toujours parfaitement réglés, leur corps droit, leur regard fixe. Ni les sentiments ni la passion ne viennent abîmer leur portrait : même lors du coït leurs visages restent « effroyablement graves et solennels » (osoroshii hodo, genshuku na hodo majime datta 恐ろしいほど、㛜肃なほどまじめだった) ${ }^{28}$ et les larmes coulent sur le visage de l'épouse « sans que le visage ne soit en rien déformé par la laideur des sanglots » (sukoshimo kyoki no minikusa ni yugandewa inainoni 少しも歔刹の醜さに歪んではいないのに) ${ }^{29}$. L'idée que le corps héroïque des personnages porte avec lui l'immobilité de la tombe, qu'il quitte les rivages organiques pour se figer dans une matière minérale ou métallique, est d'ailleurs

25. L'épouse Takeyama a un «nez d'une jolie forme » (katachi no yoi hana 形のよい 鼻, chapitre 3, p. 91), des 《lèvres au trait régulier »(tansei na kuchibiru 端正な唇, chapitre 3, p. 92), des hanches d'une 《courbe opulente » yutakana kyokusen 豊かな曲 線, chapitre 3, p. 32), le lieutenant un nez « d'une ligne admirable (biideta biryō秀で た鼻梁) et des muscles aux 《 contours distincts 》 (itaru tokoro no kinniku wa kukkirito rinkaku wo rokotsu ni arawashi いたるところの筋肉はくっきりと輪郭を露骨にあ らわし, chapitre 3, p. 94).

26. Chapitre 3, p. 92.

27. Cf. par exemple : Hasegawa Izumi, op. cit., p. 143 ; Mitsuhana Takao 光栄克 夫, Mishima Yukio-ron 三島由紀夫論 (Sur Mishima Yukio), Tōkyō, Okisekisha 沖積 舎, 2000, p. 155.

28. Chapitre 2, p. 78.

29. Chapitre 3, p. 88-89. 
exprimée à plusieurs reprises dans le texte : le lieutenant, tandis qu'il se rase devant son miroir, a ainsi l'illusion de voir son visage se détacher de lui et devenir une partie d'un monument aux morts ${ }^{30}$, plus loin il se sent enfermé dans « la cuirasse du Beau et du Juste » (bi to seigi ni yorowareta 美と正義に鎧われた $)^{31}$. Les personnages sont déjà momifiés dans l'hymne commémoratif et solennel qui leur est dédié. La photographie qui ouvre le second chapitre de la nouvelle et permet au narrateur de proposer le premier portrait-rhétorique des personnages allégorise ainsi l'ensemble du texte :

Le lieutenant, en tenue militaire, son sabre à la main gauche, son képi qu'il venait d'ôter dans la main droite, se tenait debout, droit, protégeant sa jeune épouse de manière masculine. Les traits de son visage étaient vraiment virils. Ses sourcils épais, ses prunelles grandes ouvertes, exprimaient toute la pureté, tout le courage de la jeunesse. Vêtue de son kimono à traîne blanc, la jeune mariée était d'une beauté incomparable. Dans ses yeux ronds placés sous des sourcils emplis de douceur, dans son nez fin et d'une forme agréable, dans ses lèvres potelées, se reflétaient réciproquement la noblesse et la grâce. Serrés sur un éventail, le bout de ses doigts pointait en secret sous la manche de son vêtement où ils dessinaient, alignés avec délicatesse, comme le bourgeon d'une belle-du-soir ${ }^{32}$.

Réifiés dans la mort à venir et dans les absolus qu'ils incarnent (Beauté, Bravoure, Pureté, Intégrité, etc.), les personnages ne sont pas des hologrammes en trois dimensions mais des figures immobiles, fixées sur un support commémoratif (photographie souvenir). Ils portent avec eux tout le poids du trépas comme figement ou pétrification.

30. Chapitre 3, p. 88.

31. Chapitre 3, p. 87.

32. Chapitre 2, p. 76. 


\section{Le double suicide amoureux : outre-tombe et corps vaporeux}

Si le thème et la structure énonciative du texte nous renvoient à l'épopée, celle-ci ne constitue toutefois pas l'unique canevas intertextuel sur lequel s'appuie l'auteur. Plusieurs critiques, comme Muramatsu Takeshi ${ }^{33}$ ou Tanaka Miyoko ${ }^{34}$ ont fait justement remarquer que Mishima reprend aussi, dans Yükoku, le scénario du shinjümono 心中物, les pièces de double suicide amoureux à l'origine composées pour le théâtre de poupée (bunraku 文楽) et dont les premiers grands chefsd'œuvre ont été rédigés par le célèbre dramaturge Chikamatsu Monzaemon 近 松門左衛門 (1653-1724). Il emprunte notamment aux pièces de shinjümono le thème pathétique de « la dernière fois » (saigo 最後), grand réservoir de «scène de chagrin » (shütanba 愁嘆場) : dernier regard, dernière étreinte, derniers vœux d'amours, etc. L'expression « dernière fois » apparaît d'ailleurs à de nombreuses reprises dans le troisième chapitre de la nouvelle. Les personnages sont étroitement associés à ce motif, au point de se confondre avec lui :

Le lieutenant contempla le visage de son épouse sous le sien. C'était là le dernier visage qu'il verrait en ce monde, le dernier visage d'une femme. Il l'examina minutieusement, du regard que verserait un voyageur sur les paysages splendides d'un pays dans lequel il ne reviendra pas $^{35}$.

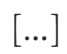

33. Muramatsu Takeshi 村松剛, cité par TAkeda Katsuhiko 武田勝彦, « Sakuhin betsu, Mishima Yukio Kenkyū-shi » 作品別三島由紀夫研究史 (Une histoire de la critique, œuvre par œuvre), in Miyoshi Yukio 三好行雄 (dir.) Mishima Yukio Hikkei 三 島由紀夫必携 (Vade-mecum Mishima Yukio), Bessatsu kokubungaku 別冊国文学 (Les lettres japonaises, numéro spécial), nº 19, Tōkyō, Gakutōsha 學燈社, 1983, p. 164.

34. TANaKa Miyoko 田中美代子, Kanshō nihon gendai bungaku $\mathrm{n}^{\circ}$ 23, Mishima Yukio 鑑賞日本現代文学23 三島由紀夫 (Anthologie commentée de la littérature japonaise contemporaine, $\mathrm{n}^{\circ} 23$ : Mishima Yukio), Tōkyō, Kadokawa shoten 角川書店, 1980, p. 162.

35. Chapitre 3, p. 88. 
Ils se retrouvèrent spontanément nus devant la lumière que diffusait le chauffage. Ils ne l'exprimèrent pas de vive voix mais leur corps, leur âme, les battements de leur cœur frémissaient à l'idée qu'il s'agissait de la dernière fois. Ces lettres « dernière fois » s'imprimèrent dans chaque recoin de leur corps d'une encre invisible ${ }^{36}$.

Plusieurs scènes de chagrin de la nouvelle Yükoku paraissent directement empruntées à Chikamatsu. La fin du chapitre trois, où sont décrites les dernières caresses qu'échange le couple, est à cet égard exemplaire. Les larmes que Reiko fait couler sur le ventre de son époux font songer à celles que le personnage de Tokubei fait couler sur le corps de son amante dans Sonezaki shinjū 曾根崎心中 (Double suicide à Sonezaki, 1703) ${ }^{37}$. Le désir que l'époux et l'épouse expriment de se contempler l'un l'autre une dernière fois pourrait évoquer plusieurs finals de shinjümono comme celui de Imamiya shinjū 今宮心中 (Double suicide à Imamiya, 1710 ${ }^{38}$. L'impossibilité dans laquelle l'épouse se trouve de retenir ses larmes au moment où elle voit son mari se préparer à la mort pourrait enfin être perçu comme un clin d'œil à l'excipit de Shinjū ten no Amijima 心中天網

36. Chapitre 3, p. 90-91.

37. Torigoe Bunzō 鳥越文蔵 (dir.), Chikamatsu Monzaemon shū 1, 近松門左衛 門集 (一) (Anthologie des œuvres de Chikamatsu Monzaemon, vol. 1), Nihon koten bungaku zenshū 日本古典文学全集 (1972), Tōkyō, Shōgakkan, 1997, p. 75. Pour la traduction française : Double suicide à Sonezaki, traduit du japonais par René SIEFFERT, in Chikamatsu Monzaemon, Les Tragédies bourgeoises, tome I, Paris, POF, 1991, p. 74. 38. Torigoe Bunzō (dir.), Chikamatsu Monzaemon shū 2 (1975), Nihon koten bungaku zenshū, Tōkyō, Shōgakkan, 1997, p. 113-114. Pour la traduction française : Double suicide à Imamiya, traduit du japonais par René Sieffert, Chikamatsu Monzaemon, Les Tragédies bourgeoises, tome III, 1992, p. 93-95. 
島 (Double suicide à Amijima pris au filet du ciel, 1721) ${ }^{39}$. À la trame épique, solennelle et héroïque, vient donc se mêler un autre cortège de topoï inspirés des arts dramaturgiques et qui soulignent la note pathétique du texte.

Dans les shinjümono, cette note pathétique est généralement associée à des jeux de clair-obscur (lumière intermittente du point du jour, de l'éclair ou d'un briquet, contraste entre les vêtements noirs et les vêtements blancs, entre la nuit et la neige ou le givre, etc.) qui contribuent à plonger le lecteur-spectateur dans une ambiance sépulcrale, comme si les personnages étaient en réalité des spectres venus rejouer pour nous, dans un décor approprié (vent qui souffle, corbeaux qui croassent, arbres décharnés, etc.), la scène, tragique et pathétique, d'un suicide déjà consommé. Donald Keene assimile ainsi le michiyuki 道行 - le chant du récitant qui, dans le troisième et dernier acte des shinjümono, accompagne les amants vers le lieu de leur suicide - à une prière de pacification (chinkon 鎮魂) de l'esprit des amants décédés rappelés pour l'occasion sur la scène ${ }^{40}$. Sur ce point le shinjūmono doit clairement beaucoup au $n \bar{o}$ 能 et nous renvoie plus globalement à l'esthétique dite du yügen 幽玄 que l'on pourrait traduire par « profondeur mystérieuse ${ }^{41} »$, idéal esthétique cultivé par les poètes et les dramaturges de l'époque médiévale $\left(\mathrm{XII}^{\mathrm{e}}-\mathrm{XV}^{\mathrm{e}}\right.$ siècle $)$ qui renvoie à l'idée de tristesse, d'ambiguïté, de mystère parfois inquiétant. Nous retrouvons cette ambiance de sépulcre dans la nouvelle Yükoku. L'écart entre la clarté que dégagent les protagonistes d'un côté (regard limpide, assimilation du lieutenant au soleil, vêtements blancs de l'épouse) et

39. Shinjū ten no Amijima (Double suicide à Amijima pris au filet du ciel), Chikamatsu jōruri shū (jō) 近松浄瑠璃集(上) (Les Jōruri de Chikamatsu, vol. 1), Nihon koten bungaku taikei 日本古典文学大系 (Anthologie des œuvres classiques du Japon), Tōkyō, Iwanami shoten, 1993, p. 385. Pour la traduction française : Double suicide à Amijima pris au filet du ciel, dans CHIKamatsu Monzaemon, Les Tragédies bourgeoises, tome IV, traduit du japonais par René Sieffert, Paris, POF, 1992, p. 213.

40. Donald Keene, “The Loves Suicides at Sonezaki” (Sonezaki Shinjū, 1703), in Shirane Haruo (dir.), Early Modern Japanese Literature: An Anthology, 1600-1900, New York, Columbia University Press, 2002, p. 243.

41. Traduction proposée par Jacqueline Pigeot : article «Shin-kokin waka-shū », in Jean-Jacques OrIgas (dir.), Dictionnaire de littérature japonaise, Paris, PUF, 2000 (1994), p. 287. 
210 Cahiers d'études japonaises n²2

l'environnement sombre dans lequel ils se déplacent (la scène se déroule de nuit, le logement est mal éclairé) donne aux personnages des airs de fantôme se déplaçant dans un décor minimaliste de mugen-nō 夢幻能 (nō d'apparition) ${ }^{42}$. Rappelons, d'autre part, que la mort des personnages de Yükoku nous est annoncée dès le prologue. Tout le texte se déploie ainsi comme une longue prière en l'honneur du couple défunt, rappelé dans le temple de leur domicile par le narrateur pour reproduire, devant le lecteur, la scène de leur dernière nuit.

Des deux personnages l'épouse est certainement celui qui offre au lecteur l'image la plus mystérieuse et la plus fantomatique. Gardienne du temple mortuaire (elle ne quitte jamais le domicile) le personnage de Reiko est clairement associée à la nuit : sa beauté est semblable à celle de « la lune après la pluie » (ugo no tsuki no yō 雨後の月のよう) ${ }^{43}$ et ses doigts sont comparés à la « belledu-soir » ${ }^{44}$ (yügao 夕顔), fleur qui éclot uniquement la nuit. Sa gracilité et son effacement ${ }^{45}$, son regard immobile ${ }^{46}$, le calme et la lenteur de ses gestes ${ }^{47}$, enfin le halo de blancheur qui l'entoure dans le noir de la cage d'escalier ${ }^{48}$ renforcent l'impression d'irréalité qui se dégage du personnage. Le portrait du lieutenant peut toutefois aussi prendre une teinte singulièrement vaporeuse :

Il approcha son visage et se rasa soigneusement devant le sombre miroir mural dont la glace était fendillée et embuée par les vapeurs d'eau chaude. Ce serait là, tel quel, son visage mortuaire. Toute trace de rasoir, déplaisante au regard, devait être à tout prix évitée. Glabre, le visage brilla de nouveau de toute sa jeunesse au point d'éclairer

42. Relèvent de cette catégorie les nô dont le personnage central n'appartient pas au monde réel. Cf. Jean-Jacques Tschudin, Histoire du théâtre classique japonais, Toulouse, Anacharsis, 2011, p. 475.

43. Chapitre 2, p. 77.

44. Chapitre 2, p. 76.

45. Chapitre 2, p. 77 ; chapitre 3, p. 91-92.

46. Chapitre 3, p. 83.

47. Chapitre 3, p. 86 ; chapitre 5, p. 107-108.

48. Chapitre 4, p. 98. 
la sombre surface sur laquelle il se réfléchissait. L'association de ce visage lumineux et plein de santé à la mort n'était pas dénuée d'une certaine élégance ${ }^{49}$.

Le portrait-rhétorique, qui ne saisit du corps que son chiffre d'or géométrique, inscrit les contours de la perfection corporelle dans un vide que signalent en creux ces apparitions fantomatiques. Sous les lignes nettes de la statue de marbre, le néant travaille, comme dans ce miroir sur lequel se dessine un visage rongé par les aplats blancs des vapeurs et coupé en deux par la surface sombre et lézardée (bibiwareta ひびわれた) qui le réfléchit. Corps vaporeux et corps héroïque se rejoignent en effet en ce qu'ils refusent tout deux le corps bien réel, avec ses imperfections et ses flaccidités que le texte semble précisément incapable de penser. Le plus frappant à cet égard est sans doute la scène du coït qui vient clore le chapitre trois. Uniquement décrit à travers des métaphores d'un style pompier - «ailes » (tsubasa 翼), 《hauteurs vertigineuses (mekurumeku takami 目くるめく高み), «porte-drapeau du bataillon lancé sur son étalon » (chōku suru rentaikishu no yō $n i$ 長駆する連隊旗手のように), 《 sommets 》 (itadaki 頂き) ${ }^{50}-$, la scène se révèle étrangement froide et n'offre qu'une image conventionnelle et abstraite de la relation sexuelle. Comme l'a bien remarqué Jerry S. Piven, nous nous trouvons ici devant une sorte de « simulacre platonique » (Platonic simulacrum) ${ }^{51}$ du coït. Dans Yükoku le corps apparaît d'abord comme déjà mort, statufié ou vaporeux, étranger à la réalité de l'existence organique. La description du seppuku marque en ce sens un bouleversement radical.

50. Chapitre 3, p. 94-95.

51. Jerry S. Piven, The Madness and Pervesion of Mishima Yukio, Westport, Praeger, 2004, p. 27. 
212 Cahiers d'études japonaises n²2

\section{La mort vivante : corps éviscéré et « esthétique de la cruauté »}

Plusieurs penseurs, philosophes comme Georges Bataille ${ }^{52}$, ou anthropologues comme Mary Douglas ${ }^{53}$, ont mis en exergue une opposition entre deux représentations antithétiques de la mort : d'un côté la mort rigide, la mort froide, pure et apaisée que symbolisent les os blanchis et le calme du tombeau; de l'autre la mort inquiétante, la mort souillée encore travaillée par l'activité organique que symbolisent les vers et le corps tuméfié qui se décompose. C'est à la mort froide et solennelle que le lecteur de Yükoku est d'abord confronté. Le corps vaporeux et les images outre-tombales disséminées dans le texte contribuent, certes à donner à celle-ci un tour parfois mystérieux, voire inquiétant. La mort reste cependant ici abstraite, limitée à ce statut de signe scripturaire auxquels les personnages, réduits à une série de lignes, tendent à être eux-mêmes assimilés. La longue scène du seppuku, placée au milieu du chapitre quatre, rompt avec ces représentations et nous introduit dans l'horreur et la violence de la mort organique. La transition est préparée et annoncée. La mort, note le narrateur au début du chapitre quatre, 《 se fait de plus en plus visible» (shiga iyoiyo genzen suru 死がいよいよ現前す る) ${ }^{54}$. «Il y aura peut-être aussi des choses difficiles à regarder 》 (migurushii koto mo arukamoshirenai 見苦しいこともあるかもしれない) ${ }^{55}$ annonce d'autre part le personnage du lieutenant à Reiko. L'écart minime entre l'expression 《 visage de mort » (shinigao 死顔 ${ }^{56}$ présente à plusieurs reprises dans le chapitre trois et 《 visage de la mort » (shi no kao 死の顔) qui apparaît au début du chapitre quatre ${ }^{57}$, résume tout le trajet qui sépare la mort comme pétrification, taxidermie qui valorise les belles formes, et la mort comme activité, incarnation qui contamine et agit sur le vivant. Le symbole le plus marquant et le plus poétique

52. Georges Bataille, L'Érotisme, Paris, Les Éditions de Minuit, 1957, p. 62-64.

53. Mary Douglas, Purity and Danger: An Analysis of Concepts of Pollution and Taboo, New York, Psychology Press, 2002 (1966).

54. Chapitre 4, p. 97.

55. Chapitre 4, p. 99.

56. Chapitre 3, p. 87 , p. 88 et p. 92.

57. Chapitre 4, p. 96. 
de ce basculement nous est sans doute fourni par les larmes de Reiko. Tandis que les premiers sanglots que verse l'épouse glissent sans laisser la moindre trace sur le visage blanc et statufié du personnage, les derniers font au contraire couler son maquillage ${ }^{58}$. Détail anodin mais porteur d'un sens profond : le masque des belles formes se désagrège. Devant la violence du seppuku le couple perd son maintien glacé. Les sécrétions corporelles envahissent la chambre, suggérant une véritable liquéfaction du corps héroïque.

La description du seppuku pourrait évoquer plus précisément la notion bataillienne de pléthore, cette croissance « impersonnelle » de la vie que l'on retrouve dans les « organes gonflés de sang » de l'animal que le sacrificateur met à nu. La « continuité organique de la vie » se dévoile alors au sein même de la discontinuité, la mort surgit et déborde comme la vie même. En s'ouvrant le ventre le personnage du lieutenant Takeyama donne à voir à sa femme (et au lecteur) une mort qui a « le rejaillissement de la vie » et une vie qui a « la lourdeur, le vertige et l'ouverture de la mort ${ }^{59} \gg$. La mort est décrite comme une poussée violente, un désordre qui jaillit 《au rythme du pouls (myakuutsu yōni hotobashitta 脈打つ ように进った ${ }^{60}$ et atteint son point d'orgue avec la poussée des intestins hors du ventre :

Lorsqu'enfin le lieutenant parvint au flanc droit, la lame maintenant légèrement à découvert, était luisante de sang et de graisse. Soudain, pris de haut-le-cœur, illâcha un cri rauque. La nausée compliquait une douleur déjà excessive. Son ventre, jusqu'alors fortement tendu, ondula subitement, la plaie devint béante et les viscères en jaillirent, tout comme si l'entaille eût dégorgée avec vigueur un mélange de vomissure et de matières excrémentielles. Semblant tout ignorer de la douleur de son propriétaire, l'intestin se glissa dehors joyeusement et se déversa entre ses cuisses, plein de santé, sous une forme si vivante qu'elle en était répugnante.

58. Chapitre 4, p. 101.

59. Georges Bataille, op. cit., p. 101-102.

60. Chapitre 4, p. 105. 
Le lieutenant baissa la tête. Il respirait péniblement, les yeux entrouverts; de sa bouche pendait un filet de bave. À ses épaules scintillait l'or des galons ${ }^{61}$.

Le ventre du personnage dont le narrateur avait mis en avant, dans le précédent chapitre, les muscles dessinés, évoquant la statuaire grecque, devient lui-même lâche, liquide. Il constitue ainsi la focale à partir de laquelle se déploient les imaginaires antithétiques du corps hérö̈que, associé à la mort monumentale et pur d'un côté; et du corps liquide et morcelé associé à la mort vivante et impure de l'autre. L'extrait évoque précisément un fantasme de la mort comme renaissance, dont plusieurs auteurs ont souligné la présence dans l'œuvre du romancier ${ }^{62}$. L'intestin, objet partiel individué et animé, est semblable à un nourrisson monstrueux qui récupère la vie de celui qui l'enfanta. Marqué à la fois par des motifs éruptifs et l'idée d'un démembrement, la scène du seppuku illustre en soi toute l'ambivalence propre à la pulsion de mort (et par extension à toute pulsion) qui implique à la fois un élan (strieb en allemand) et de l'autre une régression. En s'ouvrant le ventre, le personnage du lieutenant sépare en effet son corps en deux morceaux distincts et se dirige ainsi vers un état d'intégration moins accompli. Il concrétise ainsi cette tension vers la désintégration caractéristique de la pulsion de mort ${ }^{63}$.

Si le souci de rendre un compte exact de l'éventrement est indubitable (mention de la taille de la plaie ${ }^{64}$, des organes touchés ${ }^{65}$, de l'odeur qui imprègne

61. Chapitre 4, p. 105.

62. Pour Hélène Piralian, l'équilibre psychique de l'écrivain s'organise autour d'une mort forcluse et non symbolisée qui fait retour dans le réel comme « condition de naissance ». Cf. : Hélène Piralian, Un enfant malade de la mort: Lectures de Mishima, relecture de la paranoïa, Paris, Éditions universitaires, 1989, p. 16. Jerry S. Piven propose une lecture semblable, d'inspiration plus classiquement freudienne (op. cit., 2004, p. 51).

63. Cf. article «Pulsion de mort», in Jean Laplanche et Jean-Bertrand Pontalis, Vocabulaire de la psychanalyse, Paris, PUF, 1992 (1967), p. 371-378.

64. Chapitre 4, p. 104.

65. Chapitre 2, p. 102 et chapitre 4, p. 105. 
la pièce ${ }^{66}$, etc.) la présence de représentations qui nous renvoient à l'inconscient de l'auteur mettent à mal la dimension strictement réaliste de la scène qui prend un aspect exubérant et halluciné (personnification des intestins, geysers de sang qui se répand en quantités astronomique). Sans chercher à nier l'influence des obsessions personnelles de l'auteur, il nous paraît important d'insister sur le fait que Mishima s'appuie, ici aussi, sur un imaginaire préexistant. Le mélange de réalisme et d'outrance presque grotesque qui caractérise la scène nous renvoie en l'occurrence, comme l'a bien remarqué Noguchi Takehiko, aux arts décadents du $\mathrm{XIX}^{\mathrm{e}}$ siècle, période dominée par la culture urbaine de la ville d'Edo et qui voit éclore un art populaire bien plus violent que par le passé dont les estampes et les illustrations de Tsukioka Yoshitoshi 月岡芳年 (1839-1892), riches en scènes d'horreur tirées de faits divers, en seppuku sanglants et têtes coupées, fournissent un exemple caractéristique ${ }^{67}$. L'expression « esthétique de la cruauté » (zankoku no $b i$ 残酷の美) a été forgée par la suite pour décrire cette fascination complaisante pour la violence telle qu'elle s'exerce dans le théâtre kabuki chez des auteurs aussi célèbres que Tsuruya Nanboku 鶴屋南北 (1755-1829) et Kawatake Mokuami 河 竹黑阿弥 (1816-1893).

L'《esthétique de la cruauté » ne renvoie toutefois pas uniquement à la description outrancière de la violence. Elle est, par définition, aussi associée à l'idée d'une esthétisation de celle-ci. Le contraste chromatique entre le rouge et le blanc, déjà fréquent dans le kabuki du XVIII ${ }^{\mathrm{e}}$ siècle $^{68}$, peut notamment être considéré comme l'un des topoï plastiques des arts décadents de la fin de l'époque d'Edo. Les dramaturges du $\mathrm{XIX}^{\mathrm{e}}$ siècle jouent aussi de l'écart entre l'horreur du sujet et

66. Chapitre 4, p. 105.

67. Noguchi Takehiko, Mishima Yukio no sekai 三島由紀夫の世界 (L’univers de Mishima Yukio), Tōkyō, Kōdansha 講談社, 1968, p. 227.

68. Dragomir Costineanu, Origines et mythes du kabuki, Paris, POF, 1996, p. 360-365. 
la dimension lyrique des images qu'il suscite ${ }^{69}$. Nous retrouvons précisément ces deux éléments dans la scène du seppuku. La bichromie rouge/blanc se substitue tout d'abord aux jeux d'ombres et de lumières qui précèdent. Une goutte rouge se dépose sur le vêtement de l'épouse, puis c'est tout le bas de sa robe qui s'imprègne de carmin. Comme dans certaines estampes de Tsukioka Yoshitoshi, le sang, loin de se répandre dans un désordre brouillon et aléatoire, dessine des 《entrelacs somptueux et plein d'audace » (karei de daitan na 華麗で大胆な) sur la robe blanche de l'épouse, transformée ainsi en « robe à motifs ascendants » (susomoyō 裾模様 $)^{70}$. Cette mort esthétique suscite des images très lyriques, parfois volontairement innocentes, qui tranchent avec le réalisme et l'outrance de la scène :

La douleur se diffusait petit à petit, du fond de ses entrailles, et bientôt tout son ventre lui fit écho. C'était comme un carillon fou, comme mille cloches qu'on aurait, à chaque halètement, à chaque pulsation de son cœur, fait sonner simultanément et sous les coups desquelles tout son être chancelait. Il ne pouvait plus réprimer les gémissements que lui arrachait la souffrance. Un rapide coup d'œil lui indiqua cependant que la lame avait déjà fendu le ventre jusqu'au bas du nombril. Satisfait, il reprit courage.

69. Voir à cet égard l'introduction de Mark Oshima à la traduction en anglais de la célèbre scène Kasane 累de la pièce de kabuki Kesakakematsu naritanoriken 法懸松成田利剣 (Un pin où l'on pend et l'épée de Narita, 1823) de Tsuruya Nanboku. Mark Oshima, « Kasane », in James R. Brandon et Samuel L. Leiter (dir.), Kabuki Plays on Stage, Darkness and Desire, 1804-1864, Honolulu, University of Hawai'i Press, 2002, pp. 120-123.

70. Chapitre 5, p. 107. Le terme susomoyo 裾模様 (strictement 《 robe à motif de traîne » que nous traduisons ici par « robe à motifs ascendants ») désigne des kimono dont les motifs partent du bas du vêtement, et n'occupent que la partie inférieure par opposition au sōmoyō 総模様, kimono qui sont entièrement recouverts de motifs. Dans les susomoyō, l'effet esthétique tient donc au contraste entre deux parties de la robe, l'une vide de tout motif, l'autre pleine, avec souvent un passage progressif de l'une à l'autre 
Le sang progressait petit à petit, fidèle à sa feuille de route, jaillissant de la plaie au rythme du pouls. La natte qu'il avait face à lui était rougie par ses embruns. Dans les replis de son pantalon kaki des flaques s'étaient constituées qui se déversaient maintenant au sol. Enfin, une goutte de sang - oiselet au long cours - atterrit sur les genoux de Reiko, tout de blanc vêtue ${ }^{71}$.

Le lecteur est pris, ici, dans un balancement permanent entre des métaphores poétiques et des détails plus réalistes, contrastelui-même dédoublédansl'alternance entre les sensations auditives (d'un côté les cloches, de l'autre les gémissements) et visuelles (d'un côté les embruns et l'oiselet, de l'autre les flots mécaniques au rythme du pouls). L'écart entre la violence de la scène et la délicatesse des images qu'il suscite prend une tournure paroxystique avec l'《oiselet ( (kotori 小鳥) de sang, cliché d'innocence dont on suit, comme dans un slow motion hollywoodien, le long et lent trajet jusqu'à la robe de Reiko.

La mort vivante et l'esthétique de la cruauté jouent un rôle fondamental dans le renouvellement des stéréotypes sollicités par le texte. Les représentations figées empruntées à des genres codés comme les gunkimono ou les shinjümono entrent en collision avec un imaginaire tout à fait différent. L'épopée, dans laquelle s'enracine le scénario intertextuel du seppuku, ne proposait de l'éventrement qu'une image convenue et singulièrement éthérée. C'est «la mort sans le mourir » note ainsi Andrew Rankin à propos de la chronique guerrière Kamakura ózōshi 鎌倉大草 紙 (Le grand livre de Kamakura, 1490):

Il y a quelque chose d'artificiel dans ce rapport [épique] de suicide et d'exécutions. Un censeur pointilleux semble avoir épuré les épisodes de tout élément insupportable au regard. Bien que seize hommes se poignardent à mort, il n'y a pas de sang, pas de cri, pas d'agonie. On pourrait dire qu'il n'y pas le mourir, mais simplement la mort [...] Cette sorte d'esthétique est omniprésente dans les textes mettant en scène des samouraïs et a joué un rôle

71. Chapitre 4, p. 105-105. 
crucial pour faire émerger et promouvoir l'idéal du seppuku. Si les auteurs avaient choisi le réalisme graphique, le seppuku aurait sans doute été un phénomène de plus courte durée. Mais si la mort est intensément poétique, alors chaque homme peut aspirer à devenir un poème ${ }^{72}$.

Mishima conserve le cadre épique, monumental, associé à l'image d'une mort abstraite et inoffensive. Mais il déconstruit de l'intérieur cette image idéale de la mort en s'attardant complaisamment sur la mort organique, et en opposant volontairement deux représentations antithétiques et presque incompatibles de la mort. L'esthétique de la cruauté confère aussi au personnage de Reiko un visage très différent de celui d'épouse exemplaire dont elle semblait d'abord pourvue. Dans le dernier chapitre, son apparence vaporeuse et outre-tombale prend un tour singulièrement angoissant. La robe maculée de sang, les lèvres fardées de rouges ${ }^{73}$, elle s'attarde à l'étage où flotte déjà une odeur de putréfaction et contemple longuement, comme fascinée, le cadavre de son mari sur lequel elle finit par déposer un baiser. Yoshimura Teiji évoque à son sujet « l'épouvante attachée aux êtres inquiétants et démoniaques qui ne sont pas de ce monde » (konoyo no mononaranu ayashii mashō no sugosa ga aruこの世のものならぬ妖しい魔性 の凄さがある $)^{74}$.

\section{Collision des représentations et corps psychotique}

Dans son célèbre ouvrage L'Acte de lecture, Wolfgang Iser souligne que la compréhension globale d'un texte exige du lecteur qu'il le coordonne ${ }^{75}$. Le texte de Yükoku pose, en l'occurrence, un défi au destinataire qui doit concilier des imaginaires contraires, des normes textuelles et esthétiques qui sont placées dans

\section{Chapitre 5, p. 107.}

74. Yoshimura Teiji, Mishima ni okeru girisha 三島におけるギリシャ (La Grèce chez Mishima), in Gendai no esupuri 現代のエスプリ, op. cit., 1971, p. 106.

75. Wolfgang ISER, L'Acte de lecture, théorie de l'effet esthétique, traduit de l'allemand par Evelyne Szyncer, Bruxelles, Pierre Mardaga, 1985 (1976), p. 69-92. 
une « négation réciproque ${ }^{76}$. Les différentes images du corps qui structurent le texte renvoient certes à des imaginaires très répandus, sinon universaux, associées aux genres héroïques, aux genres fantastiques et à l'écriture fantasmatique de la mort violente, ici insérés, ainsi que nous l'avons montré, dans un cadre japonisant. Mais leur superposition dans un texte bref crée un effet de collision. La scène de l'éventrement, pourtant annoncée depuis le début du roman, constitue un tel décalage avec ce qui précède qu'elle comporte malgré tout - comme le suggèrent les réactions de nombreux critiques, déstabilisés par la violence de la description ${ }^{77}$ - quelque chose d'inattendu. Une tension s'installe que le lecteur sera incité à réduire en recherchant une nouvelle cohérence :

La contradiction neutralise le potentiel de configuration pour en faire une possibilité problématique et contestée, car elle est incapable de trouver un fondement à l'équivalence des signes. Toutefois ceci ne doit pas vouloir dire que la formation de ces configurations insuffisamment cohérentes n'a pas de sens. Au contraire la problématique va inciter le lecteur à représenter le rapport des signes par une autre configuration. Le plus souvent, le lecteur cherchera une nouvelle combinaison qui pourra remplacer celle qu'il avait trouvée tout d'abord ${ }^{78}$.

La contradiction relance l'implication du lecteur invité à apporter un nouveau code, favorisant une plus grande intégration du texte. La remarque de Yoshimura Teiji offre un exemple de relecture du texte. L'imaginaire sanglant du seppuku modifie la perception du personnage de Reiko et amène le lecteur à lui attribuer d'autres caractéristiques que celle d'épouse soumise qui lui était initialement dévolue. Aucun lien n'est toutefois établi entre les deux visages opposés de Reiko. Est-ce qu'il serait, par contraste, possible de proposer une grille

76. Ibid., p. 190.

77. Hasegawa Izumi, op. cit., 1971, p. 154-155; Mitsuhana Takao, op. cit., 2000, p. 151.

78. Wolfgang ISER, op. cit., 1985, p. 235. 
220 Cahiers d'études japonaises n²2

d'interprétation qui permette d'insérer, dans une même configuration sémantique, les représentations variées et parfois antithétiques qui traversent le texte?

Notre hypothèse est qu'il existe un point commun entre les trois types de représentations du corps et de la mort que nous avons été amené à décrire dans Yükoku : toutes trois pourraient évoquer l'univers psychotique, tel qu'il a été notamment décrit par Gisela Pankow dans L'homme et la psychose. Fréquentes sont tout d'abord, chez les sujet psychotiques, les représentations d'un « corps paralysé $\gg{ }^{79}$ qui laisse le patient dans un état de prostration. Le patient se sent écrasé sous une cuirasse qu'il souhaiterait, mais en vain, ôter ${ }^{80}$. Le corps est, plus fréquemment encore, perçu comme une « enveloppe vide ${ }^{81}$, une forme vidée de son contenu. Ces deux représentations du corps expriment la menace d'une fragmentation. Le corps n'est plus vécu comme unité, il se disperse en parties dissociées les unes des autres et indépendantes du tout qui devrait les subsumer ${ }^{82}$. Le lien s'établit naturellement entre ces trois imaginaires propres à l'univers psychotique (corps paralysé, corps vide, corps fragmenté) et les trois types de représentations du corps que nous avons décrits dans la nouvelle (corps monumental, corps vaporeux, corps éviscéré). La description, dans la scène du seppuku, de l'intestin comme un être autonome, indépendant de son « propriétaire » (aruji 主) évoque précisément la notion pankowienne de « dissociation » : une partie du corps devient le tout et perd son lien avec le reste du corps « pour réapparaître dans le monde extérieur ${ }^{83} \gg$. Les nombreuses références, dans les premiers chapitres de la nouvelle, aux contours ou à la forme du corps (sujime 筋目 ${ }^{84}$, rinkaku 輪郭 ${ }^{85}$, katachi 形 ${ }^{86}$, etc.) mériteraient alors

79. Gisela Pankow, L'Homme et sa psychose, Paris, Flammarion, 1993 (1969), p. 42.

80. Ibid., p. 49.

81. Ibid., p. 63.

82. Ibid., p. 120-121.

83. Ibid., p. 121.

84. Chapitre 3, p. 94.

85. Cf. notamment chapitre 3, p. 91-94.

86. Chapitre 2, p. 76 et chapitre 3, p. 91. 
d'être relues comme l'expression d'une angoisse de perte de mainmise sur le corps. Elles indiqueraient la menace d'un éclatement et d'un effacement des formes sous la pression d'un vide intérieur ${ }^{87}$.

La grille de lecture du corps psychotique attribue ainsi aux différentes représentations du corps qui travaillent la nouvelle une grammaire commune. Elle met en exergue l'effet de contamination (Genette) ${ }^{88}$ des intertextes et des représentations antithétiques présents dans la nouvelle : les thèmes et les imaginaires s'enrichissent mutuellement, se recoupent dans une nouvelle configuration sans pour autant perdre leurs caractères propres. Parmi les passages les plus frappants de la nouvelle il faudrait sans doute citer ceux où se lit le plus clairement la parenté de ces trois visages du corps et de la mort. Les différents imaginaires exploités par le texte sont alors réunis sur quelques lignes, composant un segment textuel d'une forte intensité, comme à la fin du chapitre quatre lorsque le lieutenant, éviscéré, est assimilé à une sorte de pantin :

Le lieutenant n'avait plus le visage d'un homme vivant. Les yeux étaient creux, la peau sèche. Ses joues, ses lèvres d'une telle beauté avaient maintenant la couleur d'une terre desséchée. Seule sa main droite, qui soutenait avec difficulté le poids du sabre, s'agitait de façon frivole, comme le bras d'une marionnette à fils, essayant de frapper la gorge avec la pointe de la lame ${ }^{89}$.

La description combine les représentations d'un corps statufié (la peau desséchée), d'un corps fantomatique (visage d'outre-tombe, yeux creux), et d'un corps dissocié (intestins séparés du corps, gestes mécaniques d'un bras qui ne lui appartient plus). Cette conjonction des représentations psychotiques fait du corps du lieutenant une sorte de caricature des portraits héroïco-épiques qui précèdent. Le corps se momifie, mais sous un jour monstrueux, qui est comme un négatif de l'imaginaire minéral qui précède : la mort asséchée mais sans la paix du monument, le geste contraint et répétitif mais sur un mode grotesque (maladresse,

87. Chez le patient psychotique, note Gisela Pankow, « chaque parcelle du corps ressenti est de la terre ferme qu'on conquiert sur le processus de la psychose », op. cit., 1993, p. 25. 88. Gérard Genette, Palimpsestes, Paris, Seuil, 1982, p. 370. 
222 Cahiers d'études japonaises n²2

absence de contrôle). Le parallèle entre ce corps plus mort que vivant et la statuaire héroïque est suggéré par le narrateur qui, évoquant les yeux, la peau, les joues et les lèvres du personnage, semble imiter, la description du corps glorieux saisi dans la perfection de ses détails.

L'exemple le plus frappant d'une conjonction des représentations psychotiques nous est sans doute fourni par l'extrait de la salle de bain cité plus haut. Nous avons souligné la solidarité qui se dégageait, dans ce passage, entre le corps héroïque d'un côté, et le corps fantomatique de l'autre. Le visage du lieutenant, qui se reflète dans le miroir sombre et embué, est à la fois irréel et luminescent, impalpable et majestueux. La suite de l'extrait souligne le lien entre l'immatérialité du corps vaporeux et le corps monumental, mais elle y associe l'idée d'une dissociation qui pourrait être perçu comme un signe avant-coureur de l'éventrement qui va suivre :

Ce serait là, tel quel, son visage mortuaire ! Ce visage, pour être plus précis, s'était à moitié détaché de son propriétaire, était devenu le visage d'un soldat mort sur un monument commémoratif. Il ferma les yeux, par curiosité. Tout était enveloppé par les ténèbres. Il n'était d'ors et déjà plus un être humain qui voit ${ }^{90}$.

Énigme insoluble, la mort, comme la pureté, est inénarrable. Plutôt que de la mort, c'est donc de son désir, de ses craintes, de ses fantasmes, note Michel Picard, que l'écrivain parle quand il évoque ce sujet ${ }^{91}$. Au moment où il conduit son personnage à imaginer sa propre mort, l'auteur semble, en l'occurrence, projeter sur la scène un condensé des représentations propres à l'univers psychotique. Le corps est en effet conçu, ici, à la fois comme vide (reflet rongé de néant), paralysé (monument aux morts) et fragmenté (une partie se détache du tout). La conjonction, sur un court fragment, de représentations antithétiques et néanmoins liées confère à ce passage une grande intensité sémantique et poétique.

90. Chapitre 3, p. 88.

91. Michel PiCARd, La Littérature et la mort, Paris, PUF, 1995, p. 60. 


\section{Conclusion}

Comme tout fait culturel majeur, la mort et l'agonie sont prises dans un réseau imaginaire préexistant, dans une série de codes qui en organisent la représentation ou le déroulement. Figurer et raconter la mort, c'est donc de se confronter à la question des stéréotypes. On peut schématiquement considérer qu'il existe, à cet égard, deux réponses : soit l'écrivain entretient l'espoir, peut-être optimiste, de s'affranchir des stéréotypes; soit il décide au contraire de les accepter avec l'ambition de les retravailler de l'intérieur. La nouvelle Yükoku de Mishima Yukio nous semble représentative de cette seconde alternative. L'auteur reprend toute une série de topoï liés à la représentation de la mort volontaire. Plusieurs strates et genres de l'histoire littéraire nationale sont ainsi amalgamés. L'objet de cette étude était de montrer précisément quels étaient ces genres et ces topoï dont s'inspire le texte, quelles images de la mort ils véhiculent, comment ils s'articulent les uns aux autres et quels effets naissent de leur rencontre.

Le cadre thématique et énonciatif de la nouvelle évoque tout d'abord les textes édifiants du passé. L'auteur reprend plus précisément le motif des derniers instants exemplaires propre aux épopées médiévales (gunkimono). La mort des héros est en l'occurrence présentée ici comme glorieuse et monumentale, une mort qu'il faut commémorer et qui est donc associée à l'idée de figement et de pétrification. La nouvelle ne saurait toutefois être réduite à son intertextualité épique. L'auteur s'inspire aussi des pièces de double suicide amoureux (shinjümono), d'abord composées pour le théâtre de poupées (bunraku) au début du XVIII ${ }^{\mathrm{e}}$ siècle. Cette seconde couche d'intertextualité, qui ajoute une note pathétique au thème héroïque du récit, nous semble indissociable d'une ambiance outre-tombale qui tient notamment au clair-obscur. À l'instar des héros tragiques des shinjümono de Chikamatsu Monzaemon, les personnages de Yükoku sont présentés comme des sortes de fantômes venus rejouer, pour les lecteurs, un suicide déjà accompli. À la mort monumentale se mêlent ainsi les représentations d'une mort fantomatique. Ces deux imaginaires ne sont pas incompatibles en ce qu' ils sont tous deux associés à l'idée d'un corps abstrait et vide. La scène du seppuku marque à cet égard un bouleversement. La mort prend ici un visage très concret, vivant et violent, associé à l'idée d'une fragmentation du corps. La sexualisation évidente de cette scène nous renvoie bien évidemment à la figure de l'auteur. Mishima s'appuie cependant 
aussi sur un imaginaire préexistant. Les deux derniers chapitres de la nouvelle évoquent en l'occurrence la notion d' « esthétique de la cruauté » (zankoku no $b i)$, fascination et esthétisation de la violence et du sang présente dans le kabuki et dans d'autres formes d'arts populaires du XIX ${ }^{\mathrm{e}}$ siècle (notamment les estampes et les livrets illustrés).

Le cadre initial du texte - le topos épique d'une mort belle et digne de louanges devient ainsi le creuset d'une rencontre entre différents imaginaires associés à la représentation de la mort volontaire et violente dans la littérature japonaise. Le minimalisme de la nouvelle, l'unité classique de temps et de lieu qui structure le récit permettent à l'écrivain de tenir ensemble ces différents imaginaires et de faire de ce texte une intéressante caisse de résonance intertextuelle. L'auteur renouvelle ainsi de l'intérieur les stéréotypes, notamment en créant un choc entre des imaginaires contraires qui finit par jeter le doute sur la signification réelle du texte. Quel statut donner à ces protagonistes qui nous sont d'abord présentés comme des héros épiques d'une grande moralité mais sont parfois décrits comme d'inquiétantes créatures avides de mort et comme venues d'outre-tombe ? Et à quel genre appartient un texte dont le cadre épique et archaïsant finit, dans la scène du seppuku, par se déliter sous l'assaut des représentations fantasmatiques que véhicule l'éviscération?

L'écart entre les deux derniers chapitres de la nouvelle et les trois premiers pourrait précisément évoquer la notion isérienne de « contradiction ». Le lecteur est ici invité à trouver un motif permettant de réunir les représentations antithétiques et de les éclairer mutuellement. Nous avons ainsi proposé une relecture du texte à travers la grille interprétative du corps psychotique. Les trois imaginaires stéréotypés sollicités par le narrateur (corps monumental de l'épopée, corps vaporeux du shinjümono, corps morcelé de l'esthétique de la cruauté) paraissent en effet étroitement indexées sur trois différents types de représentations du corps présents dans l'univers psychotique (corps paralysé, corps vide, corps fragmenté). La nouvelle Yükoku nous rappelle ainsi que la mort est sans doute le meilleur alibi que les écrivains aient à leur disposition pour parler du plus intime de leur vie, c'est-à-dire de leurs représentations inconscientes ou de leur rapport au corps. La grande réussite de Mishima est peut-être d'avoir su introduire ses représentations personnelles sous les trames entremêlées d'un complexe tissu 
intertextuel et d'avoir réussi à faire de ce texte à la fois le support d'un jeu avec les conventions littéraires et le lieu d'un investissement très personnel.

\section{Bibliographie}

\section{En français}

Barthes Roland, Sade, Fourier, Loyola, Paris, Seuil, 1971, 191 p.

Bataille Georges, L'Érotisme, Paris, Les Éditions de Minuit, 1957, 306 p.

Brisset Claire-Akiko, Brotons Arnaud, Struve Daniel, De l'épopée au Japon: Narration épique et théâtralité dans Le Dit des Heike, Paris, Riveneuve, 2011, 206 p.

Chikamatsu Monzaemon, Les Tragédies bourgeoises, tome I, Paris, POF, 1991, $301 \mathrm{p}$.

Chikamatsu Monzaemon, Les Tragédies bourgeoises, tome III, Paris, POF, 1991, $290 \mathrm{p}$.

Chikamatsu Monzaemon, Les Tragédies bourgeoises, tome IV, Paris, POF, 1992, $333 \mathrm{p}$.

Costineanu Dragomir, Origines et mythes du kabuki, Paris, POF, 1996, 477 p.

Eco Umberto, Lector in Fabula - Le rôle du lecteur ou la coopération interprétative dans les textes narratifs, Paris, Grasset, 1985 (1979), 315 p.

Genette Gérard, Palimpsestes, Paris, Seuil, 1982, 467 p.

Iser Wolfgang, L'Acte de lecture, théorie de l'effet esthétique, traduit de l'allemand par Evelyne Szyncer, Bruxelles, Pierre Mardaga, 1985 (1976), 404 p. 


\section{CIPANGO}

226 Cahiers d'études japonaises n²2

Labarthe Judith, L'Épopée, Paris, Armand Colin, 2006, 360 p.

Laplanche Jean, Pontalis Jean-Bertrand, Vocabulaire de la psychanalyse, Paris, PUF, 1992 (1967), $523 \mathrm{p}$.

Mishima Yukio, La Mort en été, traduit de l'anglais par Dominique Aury, Paris, Gallimard, 1983, 320 p.

Pankow Gisela, L'Homme et sa psychose, Paris, Flammarion, 1993 (1969), 305 p.

PiCARD Michel, La Littérature et la mort, Paris, PUF, 1995, 193 p.

Piralian Hélène, Un enfant malade de la mort: Lecture de Mishima, relecture de la paranoïa, Paris, Éditions universitaires, 1989, 128 p.

Sieffert René, Le Dit des Heiké, traduit du japonais par René Sieffert, Lagrasse, Verdier, 2012 (1976), 855 p.

Tschudin Jean-Jacques, Histoire du théatre classique japonais, Toulouse, Anacharsis, 2011, $506 \mathrm{p}$.

\section{En japonais}

HaSegaWa Izumi 長谷川泉, «Yükoku, Töka no kiku, Eirei no koe ni furete 》 『憂国』、『十日の菊』、『英霊の聲』に触れて $(\mathrm{Au}$ sujet $\mathrm{de}$ Patriotisme, Le Chrysanthème du dernier jour et La Voix des mânes héroïques), Gendai no esupuri 現代のエスプリ, nº 48, février 1971, p. 138-157.

Mishima Yukio 三島由紀夫, Eirei no koe 英霊の聲 (La voix des mânes héroïques), Tōkyō, Kawade shobō 河出書房, 2005(1966), 261 p + 7 p.

Mitsunana Takao 光栄克夫, Mishima Yukio-ron 三島由紀夫論 (Sur Mishima Yukio), Tōkyō, Okisekisha 沖積舎, 2000, 261 p. 
SATō Hideaki 佐藤秀明, Mishima Yukio no bungaku 三島由紀夫の文学 (La littérature de Mishima), Tōkyō, Shironsha試論社, 2009, 539 p.

TAKEDA Katsuhiko 武田勝彦, «Sakuhin betsu, Mishima Yukio Kenkyūshi »作品別 三島由紀夫研究史 (Une histoire de la critique, œuvre par œuvre), in Mryoshi Yukio 三好行雄 (dir.), Mishima Yukio Hikkei 三島由 紀夫必携 (Vade-mecum Mishima Yukio), Bessatsu kokubungaku 別冊国文 学 (Les lettres japonaises, numéro spécial), nº 19, Tōkyō, Gakutōsha 學燈 社, 1983, $220 \mathrm{p}$.

TanaKa Miyoko 田中美代子, Kanshō Nihon gendai bungaku 23, Mishima Yukio Kanshō Nihon gendai bungaku, Mishima Yukio 鑑賞日本現代文学 23 三 島由紀夫 (Anthologie commentée de la littérature japonaise contemporaine, $\mathrm{n}^{\circ} 23$ : Mishima Yukio), Tōkyō, Kadokawa shoten 角川書店, 1980, 429 p.

Torigoe Bunzō 鳥越文蔵 (dir.), Chikamatsu Monzaemon shū 1 近松門左 衛門集 (一) (Anthologie des œuvres de Chikamatsu Monzaemon, vol. 1), Nihon koten bungaku zenshū 日本古典文学全集, Tōkyō, Shōgakkan 小学 館, 1997 (1972), 601 p.

Torigoe Bunzō 鳥越文蔵 (dir.), Chikamatsu Monzaemon shū 2 近松門左 衛門集 (二) (Anthologie des oeuvres de Chikamatsu Monzaemon, vol. 2), Nihon koten bungaku zenshū 日本古典文学全集, Tōkyō, Shōgakkan, 1997 (1975), $637 \mathrm{p}$.

Yoshimura Teiji 吉村貞司, «Mishima ni okeru girisha »三島におけるギリ シャ (La Grèce chez Mishima), in Hasegawa Izumi, Gendai no esupuri 現代 のエスプリ, nº 48, Tōkyō, Shibundō, 1971, p. 96-107.

\section{En anglais}

Brandon James R., Leiter Samuel (eds), Kabuki Plays on Stage, Darkness and Desire, 1804-1864, Honolulu, University of Hawai'i Press, 2002, 405 p. 
228 Cahiers d'études japonaises n²2

Douglas Mary, Purity and Danger: An Analysis of Concepts of Pollution and Taboo, New York, Psychology Press, 2002 (1966), 203 p.

Garcin Thomas, "Representation of Death and Topoi in Mishima Yukio's Yükoku (Patriotism)", in Teodorescu Adriana (ed), Death Representation in Literature, Forms and Theories, Newcastle-upon-Tyne, Cambridge Scholars Publishing, 2015, pp. 228-245.

McCullough Helen Craig, Yoshitsune: A $15^{\text {th }}$ Century Japanese Chronicle, Stanford, Stanford University Press, 1966, 377 p.

Mishima Yukio, Patriotism, traduit du japonais par SARgent Geoffrey W., Death in Midsummer and Other Stories, New York, New Directions Publishing, 1966, pp. 93-118.

Piven Jerry S., The Madness and Perversion of Yukio Mishima, Westport, Praeger, 2004, $273 \mathrm{p}$.

Rankin Andrew, Seppuku: A History of Samurai Suicide, Tōkyō, Kodansha International, 2011, $255 \mathrm{p}$.

SHIllony Ben-Ami, Revolt in Japan: The Young Officers and the February 26, 1936 Incident, Princeton, Princeton University Press, 1973, 262 p.

Shirane Haruo (ed),Early Modern Japanese Literature: An Anthology, 1600-1900, New York, Columbia University Press, 2002, 1026 p.

Résumé : La nouvelle Yūkoku (Patriotisme, 1961) décrit le suicide rituel (seppuku) du lieutenant Takeyama Shinji et de son épouse Reiko lors de la tentative de putsch ultranationaliste du 26 février 1936. L'arrière-plan idéologique de la nouvelle, l'évidente pulsion de mort qui anime les personnages et la place prépondérante accordée à la description de l'éventrement ont amené la plupart 
des commentateurs à interpréter ce texte sous un angle biographique et/ou thématique. Mishima ne se contente toutefois pas, dans Yükoku, de broder sur ses propres fantasmes et de jouer de façon littéraire avec ses fétiches politiques. À travers le motif de la mort volontaire, il retravaille aussi des figures très présentes dans la littérature nationale. L'objet de cette étude sera de montrer quels sont les topoï présents dans Yükoku et de voir comment ils s'articulent les uns aux autres. Nous proposerons, à l'issue de cette analyse, une relecture de la nouvelle à travers la grille interprétative du corps psychotique qui offre un dénominateur commun à ces représentations hétéroclites.

Abstract: The novella Yükoku (Patriotism, 1961) recounts Lieutenant Takeyama Shinji's and his wife's ritual suicide (seppuku) following the ultranationalist attempted Coup of February 26, 1936. The text's ideological background, the character's obvious death wish, and the long and detailed depiction of the lieutenant's disembowelment have led most commentators to focus on the novella's thematic and/ or biographical aspects. Yet in Yūkoku, Mishima does not merely elaborate on his own fantasies and political fetishes; Mishima uses the theme of voluntary and violent death, as a ways to toy with Japanese literary topoi. The purpose of this article is to list some of these literary motifs and to show how Mishima combines them. By focusing on the representation of the psychotic body, in the last part of my analysis, I offer a new interpretative framework which links the heterogeneous representations present in the novella.

Mots-clés : Mishima Yukio, Topoï et stéréotypes, Seppuku, Théories de la réception

Keywords: Mishima Yukio, Topoi, Violent Death, Psychotic body, Readerresponse criticism

$$
\text { キーワード: 三島由紀夫, ステレオタイプ, 切腹, 読者反応批評 }
$$


